\title{
Modeling and simulation for low-frequency vibration energy harvesting based on piezoelectric unimorph cantilever beam
}

\author{
Binjie Song ${ }^{1}$, Jianhai $\mathrm{Yue}^{1}$ and Zhunqing $\mathrm{Hu}^{1}$ \\ ${ }^{1}$ School of Mechanical, Electronic and Control Engineering, Beijing Jiaotong University, No.3 Shangyuancun Haidian District, 100044 , \\ Beijing, China
}

\begin{abstract}
In order to solve the problem of sustainable energy supply for low-power electronic products used in low-frequency vibration environment, the mathematic model was established based on the theory of piezoelectricity and Euler-Bernoulli beam. Also, the effects of different parameters of PZT unimorph beams such as the length, width, and tip mass on generating capacity were studied by FEM. The results show that the energy harvester with PZT unimorph beam and tip mass is suitable for low-frequency vibration environment. Increasing the length or reducing the width of the beam can significantly lower the first-order modal frequency of energy harvester when other conditions remain the same. Within certain range, the firstorder modal frequency of the beam also gradually reduced as the tip mass increasing. When the size of the PZT unimorph beam is $60 \times 60 \times 0.33 \mathrm{~mm}$, the tip mass is $8.92 \mathrm{~g}$ and an exciting force of $0.01 \mathrm{~N}$ is applied to it along $z$ axis, an output of $8.1 \mathrm{~V}$ can be obtained. Meanwhile, the PZT unimorph beam is under the first vibration mode and the resonant frequency is $16.296 \mathrm{~Hz}$.
\end{abstract}

\section{Introduction}

With the development of MEMS technology, wide application of small electronic components and Wireless Sensor Networks (WSNs), many disadvantages of the traditional way using chemical battery as the main energy supply have been revealed, one of the biggest defects is the short service life, which means that batteries are difficult to fundamentally solve the problem of providing sustainable energy supply [1]. In recent years, the technology of collecting vibration energy from ambient environment and transforming it into usable electricity has been a research hot-spot [2,3].Besides, the research on the power generator based on vibration is trending to three common orientations: piezoelectric power generator, electrostatic power generator and electromagnetic power generator. [5,6], compared with the other two methods, the piezoelectric power generator based on vibration has long life, simple in structure, easy to miniaturize, no electromagnetic interference and clean environment. It can provide and meet the electric energy demand for low power electronic products and WSNs working in low-frequency vibration ambient environment. Therefore, the research on piezoelectric power generator has drawn an increasing attention [7].

Previous studies have shown that the power generating capacity of energy harvester with PZT beams depends mainly on the structural parameters of the PZT beams and the vibration energy in ambient environment $[8,9]$. Generally, the vibration energy is very limited and the frequency is relatively low in the environment. Under this circumstances, in order to make the piezoelectric energy harvester has good capacity to meet the power demand of small electronic components and WSNs working in low-frequency vibration environment $(10-30 \mathrm{~Hz})$, we must adjust the structure and shape parameters of the piezoelectric vibrator to reduce the first-order modal frequencies. Most studies have shown that the PZT beam has a relatively larger power density in low-frequency vibration environment, and can obtain larger energy output [10]. Thus, through the establishment of mathematic model and FEM, the paper studied the influence on the first-order modal frequency and power generating capacity of the piezoelectric energy harvester, when the PZT beams has different length, width and tip mass with the aim of better meeting the requirements for energy supply using in lowfrequency vibration ambient environment.

\section{Mathematic model}

The PZT unimorph beam is composed of metal substrate and piezoelectric layer, and these two layers are stuck together. Figure 1 shows the structural schematic diagram of the PZT unimorph beam. In the Cartesian coordinate system, we take the length direction as $x$ axis and take the vertical direction as $z$ axis.

When the PZT beam is driven by external excitation, the bending deformation along the $z$ direction will appear. Usually, the ratio of length to thickness of the PZT beam is very large, so that the effects of shear deformation and rotary inertia are ignored. The bending deformation in the PZT beam will cause the change of stress and strain in piezoelectric body. Based on the 
direct piezoelectric effect, the piezoelectric body will be polarized on the upper and lower surfaces, and free charge is generated, thus the voltage output is formed between the two surfaces.

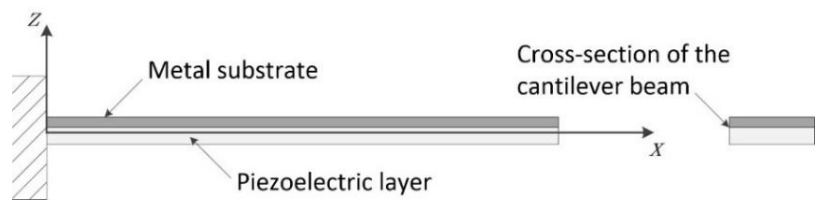

Fig 1. Structure schematic diagram of the PZT unimorph beam

We respectively use $L, w$ and $h$ to indicate the length, width and total thickness of the PZT unimorph beam. The subscript $p$ and $m$ stand for piezoelectric body and metal substrate. The ratio of $h_{m}$ and $h$ is defined as thickness ratio $\alpha$, it is obvious that $\alpha=h_{m} / h$ and the thickness of the piezoelectric layer is $h_{p}=(1-\alpha) h$.

When a force is applied at the free end of the beam, the stress and strain generated in piezoelectric layer are obviously functions of $\alpha$. According to the elastic theory of materials, the relationship between the stress and strain can be given as [11]:

$$
\begin{aligned}
& T_{p}=E_{p}\left(S_{1}-g_{31} D_{3}\right) \\
& E_{3}=-g_{31} T_{p}+\beta_{33}^{T} D_{3}
\end{aligned}
$$

where, $S_{1}=-\rho z$ and $T_{p}$ are the strain and stress generated in $x$ direction, $E_{p}$ and $g_{31}$ are the young's modulus and piezoelectric voltage constant of the piezoelectric material, $D_{3}$ is the electric displacement in $z$ direction, $E_{3}$ is the electric field strength in $z$ direction, $\beta_{33}^{T}=1 / \varepsilon_{33}^{t}$ is the dielectric impermeability, and $\varepsilon_{33}^{t}$ is the dielectric constant.

Because the metal substrate and piezoelectric body are different materials, the neutral layer of the PZT unimorph beam may not be the geometric center layer. Therefore, in this paper, the location of the neutral layer is determined by Euler-Bernoulli bean [4]. The PZT unimorph beam will bend along its neutral layer when an external force acts on the free end of the beam, in this situation, the distance between the top surface of the piezoelectric layer to the neutral layer can be written as:

$Z_{S}=\frac{E_{p} \frac{h_{p}^{2}}{2}+E_{p} h_{m}\left(h_{p}+\frac{h_{m}}{2}\right)}{E_{p}+h_{p}+E_{m} h_{m}}=\frac{h}{2} \cdot \frac{(1-\alpha)^{2}+\alpha \beta(2-\alpha)}{1-\alpha+\alpha \beta}$

where, $E_{m}$ is the young's modulus of metal substrate, $\beta=E_{m} / E_{p}$ is the elastic modulus ratio.

When an external force acts on the free end of the beam, the moment equation is given in [12] as:

$$
M=n \int_{0}^{w} \int_{Z_{S}-h_{p}}^{Z_{S}} z T_{p} \mathrm{~d} y \mathrm{~d} z+\int_{0}^{w} \int_{Z_{S}-h}^{Z_{S}-Z_{p}} z T_{m} \mathrm{~d} y \mathrm{~d} z=(x-L) F
$$

where $n$ is the number of piezoelectric layer, $T_{m}=E_{m} S_{1}$ is the stress in the metal substrate along $x$ direction.

From formula (1) and (4) can obtain equation:

$$
\begin{aligned}
& \int_{0}^{w} \int_{Z_{S}-h_{p}}^{Z_{S}} z E_{p}\left(S_{1}-g_{31} D_{3}\right) \mathrm{d} y \mathrm{~d} z+ \\
& \int_{0}^{w} \int_{Z_{S}-h}^{Z_{S}-Z_{p}} z E_{m} \mathrm{~S}_{1} \mathrm{~d} y \mathrm{~d} z=(x-L) F
\end{aligned}
$$

By $S_{1}=-\rho z$ and the formula (3) and (5), the curvature radius of the beam can be obtained as:

$$
\begin{aligned}
& \rho=-\frac{6}{A E_{p} w h^{3}} \\
& {\left[2(1-\alpha+\alpha \beta)(x-L) F+\alpha(1-\alpha) \beta w h^{2} g_{31} E_{p} D_{3}\right]}
\end{aligned}
$$

and the coefficient $A$ can be given as:

$$
A=\alpha^{4}(1-\beta)^{2}-2 \alpha\left(2 \alpha^{2}-3 \alpha+2\right)(1-\beta)+1
$$

The electric field strength can be obtained by combining (6) with (2), by integrating the electric field strength with $z$ and then voltage can be expressed as:

$$
\begin{aligned}
& V=\iint_{Z_{S 1}-h_{p}}^{Z_{S 1}} E_{3} \mathrm{~d} z= \\
& \frac{1-\alpha}{8 A w h}\left[6 \alpha \beta g_{31}(L-x) F+\frac{B w h^{2} \beta_{33}^{T} D_{3}}{1-\alpha+\alpha \beta}\right]
\end{aligned}
$$

The coefficient $B$ and $k_{31}^{2}$ can be written as:

$$
\begin{gathered}
B=A(1-\alpha+\alpha \beta)\left(1+k_{31}^{2}\right)-3 \alpha^{2}(1-\alpha) \beta^{2} k_{31}^{2} \\
k_{31}^{2}=E_{p} g_{31}^{2} l \beta_{33}^{T}
\end{gathered}
$$

The formula (8) can be reduced to:

$$
\begin{aligned}
& D_{3}=\frac{(1-\alpha+\alpha \beta)}{(1-\alpha) \beta_{33}^{T} B w h^{2}} \\
& {\left[6 \alpha(1-\alpha) \times \beta g_{31}(x-L) F+A h w V\right]}
\end{aligned}
$$

The charge generated on the piezoelectric electrode is obtained by integrating the electric displacement to the area. As the electrode is on the equal potential surface, so the charge generated on the piezoelectric surface is:

$$
\begin{aligned}
& Q=\int_{0}^{w} \int_{0}^{L} D_{3} \mathrm{~d} y \mathrm{~d} x= \\
& \frac{(1-\alpha+\alpha \beta) L^{2}}{\beta_{33}^{T} B h^{2}}\left[-3 \alpha \beta g_{31} F+\frac{A w h V}{(1-\alpha) L}\right]
\end{aligned}
$$

The last formula is a general formula about the charge when external force and self-excited electric field of the PZT unimorph beam both exist. As for piezoelectric power generator, the impacts of voltage generated by its own to the power generator are ignored, 
which means that $V=0$. Therefore the electric charge generated only by the external excitation can be expressed as:

$$
Q_{g}=-\frac{3 \alpha \beta(1-\alpha+\alpha \beta) g_{31} L^{2}}{\beta_{33}^{T} B h^{2}} F
$$

According to the relation between charge and voltage: $Q=C V$ and formula (12), the free capacitance of the piezoelectric vibrator is obtained as:

$$
C_{f}=\frac{(1-\alpha+\alpha \beta) A w L}{(1-\alpha) \beta_{33}^{T} B h}
$$

The open circuit voltage can be given according to the formula (13) and (14).

$$
V_{g}(F)=-\frac{3 \alpha(1-\alpha) \beta g_{31} L}{A w h} F
$$

At last, on the basis of formula: $U_{g}=Q_{g} V_{g} / 2$, the $U_{g}$ can be written as:

$$
U_{g}=\frac{9(1-\alpha)(1-\alpha+\alpha \beta) \alpha^{2} \beta^{2} k_{31}^{2} L^{3}}{2 A B E_{p} w h^{3}} F^{2}
$$

\section{Finite element analysis}

\subsection{The establishment of finite element model}

In order to fit the low-frequency vibration environment, we establish the finite element model of the PZT unimorph beam with tip mass. The piezoelectric body is PZT-5H, which has high electro-mechanical coupling coefficient, high piezoelectric strain coefficient, nonaging and large time constant. It is suitable to be used as a transducer [13]. The metal substrate is made of phosphorus bronze, which has large modulus of elasticity and strong fatigue resistance, can bear larger deformation. The structure and material parameters of the piezoelectric vibrator with tip mass are as follows:

Table 1. Structure and material parameters of the PZT unimorph beam

\begin{tabular}{|l|c|c|}
\hline Structure and material & PZT- & Metal \\
\hline Length of the beam, $(\mathrm{mm})$ & 60 & 60 \\
\hline Width of the beam, $(\mathrm{mm})$ & 10 & 10 \\
\hline Thickness of the beam, $(\mathrm{mm})$ & 0.22 & 0.11 \\
\hline Density of the materials, $\left(\mathrm{kg} / \mathrm{m}^{3}\right)$ & 7500 & 8920 \\
\hline Young's modulus, $(\mathrm{Gpa})$ & 5.6 & 106 \\
\hline Poisson's ratio & & 0.35 \\
\hline
\end{tabular}

The electrical boundary condition of the PZT unimorph beam is open connection. The elastic stiffness coefficient matrix $\left(\vec{c} \times 1010 \mathrm{~N} / \mathrm{m}^{2}\right)$, piezoelectric stress constant matrix $\left(\vec{e} \times C / m^{2}\right)$ and relative dielectric constant matrix $(\vec{\varepsilon})$ of PZT-5H are respectively [13]:

$$
\begin{gathered}
{[\vec{c}]=\left[\begin{array}{cccccc}
12.6 & 7.95 & 8.41 & 0 & 0 & 0 \\
7.95 & 12.6 & 8.41 & 0 & 0 & 0 \\
8.41 & 8.41 & 11.7 & 0 & 0 & 0 \\
0 & 0 & 0 & 2.35 & 0 & 0 \\
0 & 0 & 0 & 0 & 2.30 & 0 \\
0 & 0 & 0 & 0 & 0 & 2.30
\end{array}\right]} \\
{[\vec{e}]=\left[\begin{array}{ccc}
0 & 0 & -6.5 \\
0 & 0 & -6.5 \\
0 & 0 & 23.3 \\
0 & 0 & 0 \\
0 & 17.0 & 0 \\
17.0 & 0 & 0
\end{array}\right][\vec{\varepsilon}]=\left[\begin{array}{ccc}
1700 & 0 & 0 \\
0 & 1700 & 0 \\
0 & 0 & 1470
\end{array}\right]}
\end{gathered}
$$

The finite element analysis of the PZT unimorph beam is a coupled field analysis. The coupled field analysis process usually consider the cross effect and interaction of two or more engineering physical fields. In this paper, direct coupling method is adopted. The PZT$5 \mathrm{H}$ adopts Solid5 piezoelectric coupling unit, the metal substrate and tip mass adopt Solid45 entity unit. The influence of the bonding layer is neglected and it is assumed that the force and displacement between PZT$5 \mathrm{H}$ layer and metal substrate are continuous [14]. The FEM is shown in figure 2:

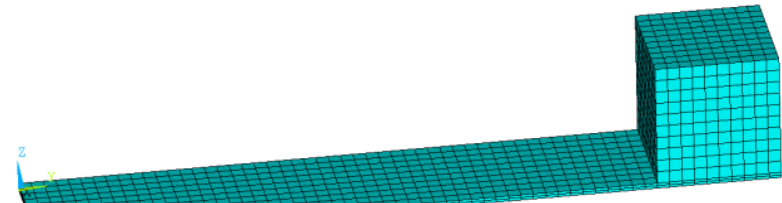

Fig 2. FEM of the PZT unimorph beam with tip mass

\subsection{Modal analysis}

In order to study the factors that influence the power generating capability of the piezoelectric energy harvester, the impacts of different length, width and tip mass of the cantilever beam on the modal frequency of piezoelectric vibrator is studied respectively.

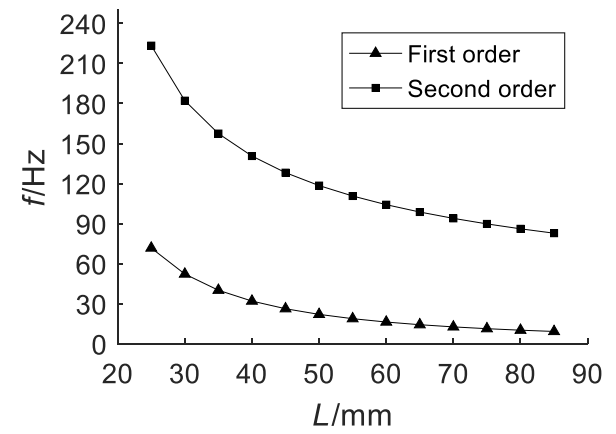


Fig 3. Variation of the PZT unimorph beam's first and second order modal frequencies with the length of the beam

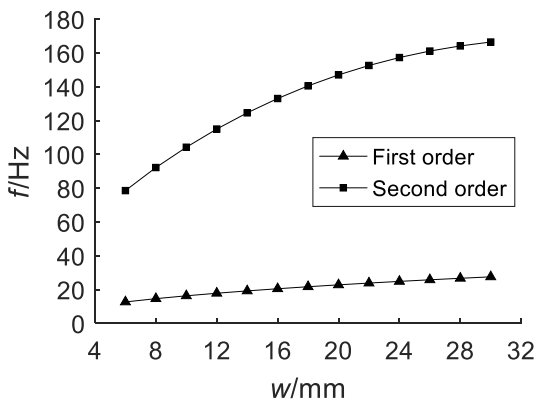

Fig 4. Variation of the PZT unimorph beam's first and second order modal frequencies with the width of the beam

The figure 3 shows that with the length of the cantilever beam increase, the first and second order modal frequencies are reduced. When the length of the beam is over $70 \mathrm{~mm}$, its effect gradually becomes weaker and weaker. Besides, we can see from the figure 4, with the width of the beam increasing, the first and second order modal frequencies are increased too.

What is shown in figure 5 is the trend of first and second order modal frequencies of the beam when the tip mass changed only. We can find that with the increase of tip mass, the resonant frequencies of first vibration mode are also gradually reduced.

According to the study, when the length is $60 \mathrm{~mm}$ ( $L=60 \mathrm{~mm})$, the width is $10 \mathrm{~mm}(w=10 \mathrm{~mm})$ and the tip mass at the end of the beam is $8.92 \mathrm{~g}(M=8.92 \mathrm{~g})$, the frequency at first vibration mode is $16.296 \mathrm{~Hz}$.

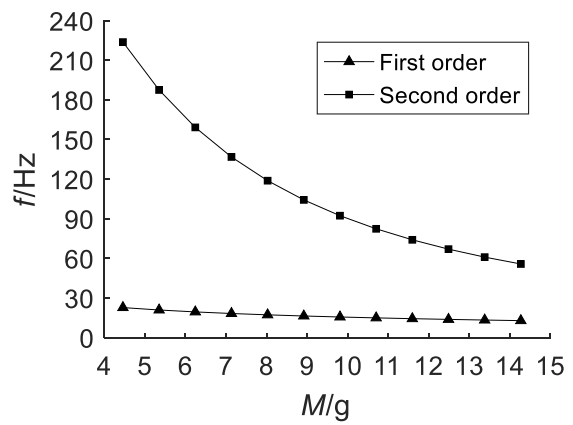

Fig 5. Resonant frequencies of the PZT unimorph beam under first and second vibration mode with the variation of the tip mass

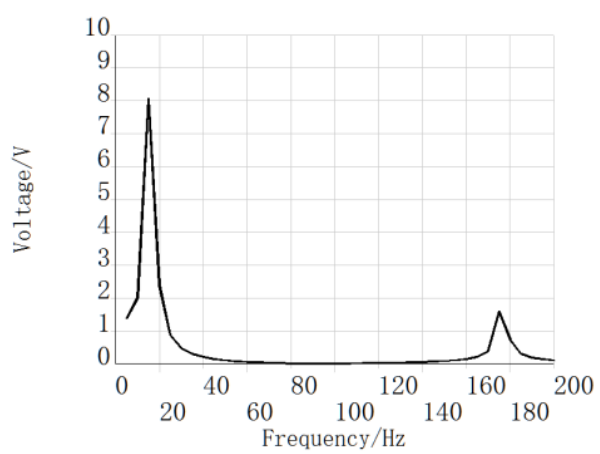

Fig 6. Relation between the voltage output and vibration frequencies

\subsection{Harmonic response analysis}

Harmonic response analysis was carried on when an external exciting force of $0.01 \mathrm{~N}$ is applied in $z$ direction on the tip mass. In ANSYS, the analysis frequency ranging from 0 to $200 \mathrm{~Hz}$, the voltage of lower PZT surface was set to 0 , and the voltage on the upper surface of the PZT layer was coupled. Through the simulation, we can get the voltage output from the coupling point on top surface of the PZT beam. What is shown in figure 6 is the relation between voltage output and vibration frequencies.

We can see from figure 6 , the peak voltage of firstmodal frequency is about $8.08 \mathrm{~V}$, and because the second vibration mode of the PZT unimorph cantilever is distortion, the polarization of PZT layer along $z$ axis is very small, which make the output voltage here is almost zero. Besides, the peak voltage output of the thirdvibration mode is $1.65 \mathrm{~V}$.

\section{Conclusion}

In this paper, the energy harvester designed which based on the PZT unimorph beam can effectively convert the mechanical vibration energy into electrical energy in low-frequency vibration environment. The length, width and tip mass of the beam have different effect on the conversion ability. When the size of the PZT unimorph beam is $60 \times 60 \times 0.33 \mathrm{~mm}$, the tip mass is $8.92 \mathrm{~g}$, the firstorder modal frequency can be as low as $16.296 \mathrm{~Hz}$. When an external exciting force of $0.01 \mathrm{~N}$ is applied in $z$ axis at the tip mass at this time, the peak voltage output of the piezoelectric energy harvester at the firstmodal is $8.08 \mathrm{~V}$, which can better meet the power requirement of small electronic components using in low-frequency vibration ambient environment. In practical applications, all the above parameters can also be optimized according to different ambient environment.

\section{Acknowledge}

The study is supported by Technology Division of China Railway (2017J004-H).

\section{References}

1. Shaikh F K and Zeadally S 2016 Energy harvesting in wireless sensor nesecondrks: A comprehensive review J. Renewable \& Sustainable Energy Reviews. 55 1041-54

2. P Glynne-Jones, M J Tudor, S P Beeby and N M White 2004 An electromagnetic vibration-powered generator for intelligent sensor systems J. Sens. Actuator. A: Phys. 110 344-49

3. D Mallick, A Amann and S Roy 2015 A nonlinear stretching based electromagnetic energy harvester on FR4 for wideband operation J. Smart Mater. Struct. 24(1) 15013-026

4. Mitcheson P D, Miao P, Stark B H, Yeatman E M, Holmes A S and Green T C 2004 MEMS 
electrostatic micropower generator for low frequency operation J. Sensors \& Actuators A Physical. 115(2-3) 523-29

5. Sodano H A, Park G, Leo D J and Inman D J 2003 Use of piezoelectric energy harvesting devices for charging batteries C.Smart Structures and Materials 2003 Conference. 101-08

6. Wang S, Lam K H, Sun C L, Kwok K W, Chan H L and Guo M S 2007 Energy harvesting with piezoelectric drum transducer J. Applied Physics Letters. 90(11) 799

7. Cheng G M, Pang J Z, Tang K H Yang Z G, Ping Z and Kan J W 2007 Development of measuring system for electricity generating capacity of piezoelectric ceramics J. Journal of Jilin University

8. Roundy S, Wright P K and Rabaey J 2003 A study of low level vibrations as a power source for wireless sensor nodes J. Computer Communications 26(11) 1131-44

9. Jiang S, Li X, Guo S, Hu Y, Yang J and Jiang Q 2005 Performance of a piezoelectric bimorph for scavenging vibration energy J. Smart Materials \& Structures. 14(4) 769

10. Jiang S N, Guo S H and Li X F 2012 Performance analysis for a unimorph cantilever piezoelectric harvester J. Journal of Vibration \& Shock. 31(19) 90-94

11. Greve D W and Oppenheim I J 2003 Energy scavenging for sensor applications using structural strains J. Proceedings of SPIE - The International Society for Optical Engineering

12. Kan J W, Tang K. H, Wang S Y, Yang Z. G, Jia J and Zeng P 2008 Modeling and simulation of piezoelectric cantilever generators J Optics \& Precision Engineering 16(1), 71-75

13. Luan G D, Zhang $\mathrm{J}$ and Wang $\mathrm{R}$ Q 2005 Piezoelectric Transducers and Arrays ed Wang Y and Sun Y (Peking University Press) chapter $3 \mathrm{pp}$ 75-102

14. Peter Koh ke 1998 Ansys Coupled-Field Analysis Guide, Release5. Ansys Inc 